\title{
Cultural adaptations of psychological treatments for depression are mostly based on implementation rather than content
}

\author{
Neil Krishan Aggarwal
}

Columbia University Medical Center, New York State Psychiatric Institute, New York, New York, USA; aggarwa@nyspi.columbia.edu

\section{WHAT IS ALREADY KNOWN THIS TOPIC?}

The WHO considers psychological interventions to be first-line treatments for mild depression and effective in combination with antidepressants for moderate and severe depression. ${ }^{1}$ However, interventions are often developed in populations from Europe and the USA. Little is known about how such treatments are adapted for minority populations in Western societies or for populations elsewhere.

\section{WHAT DOES THIS PAPER ADD?}

- Cultural adaptations of psychological treatments follow the Medical Research Council methodology to develop complex interventions, ${ }^{2}$ when described.

- Most adaptations involve replacing technical terms with local expressions, adding culturally acceptable treatment practices, involving family members, understanding patient illness models and simplifying treatments such as homework.

- Adaptations usually improve implementation rather than revising core theoretical principles. For example, phases and techniques of psychological treatments were not adapted, but technical terms for illness or exercises were replaced with local idioms.

\section{LIMITATIONS}

- The authors do not define culture at all, overlooking that different cultural factors are responsible for misunderstandings in diagnosis and treatment planning across societies. ${ }^{3}$ For example, race is a marker of cultural difference in the USA, compared to immigration status in Europe or language in South Asia. ${ }^{3}$

- The databases used have been critiqued by the same authors for publication bias towards studies from high-income countries. Region-specific databases could have been used. ${ }^{4}$ Also, there is a significant delay between the search (December 2011) and manuscript submission (March 2013).

- More information is needed on whether the clinicians delivering these interventions find such adaptations feasible, acceptable, useful or sustainable in practice.

\section{WHAT NEXT IN RESEARCH?}

Future studies should test the efficacy of culturally adapted psychological treatments versus non-adapted treatments in matched patient samples. This design would clarify differences in treatment response based on the intervention versus culturally competent therapists. Descriptive studies are needed to clarify how therapists innovate by describing how clinicians decide between fidelity and adaptation in treating diverse patients. Finally, effects of correlated variables such as socioeconomic status and literacy level should be disentangled. Treatments may have required adaptation due to the educational levels of study participants, for example, replacing biomedical terms with colloquial expressions.

\section{DO THESE RESULTS CHANGE YOUR PRACTICES AND WHY?}

These results will change my practice by explaining how to adapt interventions - such as using patient expressions, simplifying treatments and incorporating safe cultural practices within treatment planning - to improve their feasibility and acceptability with diverse patient groups. My practice in New York City consists of people from multiple race and ethnicities for which an adapted treatment based on one cultural group would be irrelevant. The value of this review is in reminding clinicians that we belong to a professional culture replete with biomedical concepts and vocabularies that is distinct from the culture of patienthood. The greatest cultural adaptation may be in translating biomedical science into understandable treatment plans accepted by our patients rather than content tailored for any specific cultural group.

Competing interests None.

doi:10.1136/eb-2014-101928

\section{REFERENCES}

1. World Health Organisation. MhGAP intervention guide for mental, neurological and substance use disorders in non-specialized health settings: mental health gap action programme (mhGAP). Geneva: WHO, 2010.

2. Craig P, Dieppe P, Macintyre S, et al. Medical Research Council Guidance. Developing and evaluating complex interventions: the new Medical Research Council guidance. BMJ 2008;337:a1655.

3. Kirmayer LJ, Minas $\mathbf{H}$. The future of cultural psychiatry: an international perspective. Can J Psychiatry 2000;45:438-46.

4. Aggarwal NK, Balaji M, Kumar S, et al. Using consumer perspectives to inform the cultural adaptation of psychological treatments for depression: a mixed methods study from South Asia. J Affect Disord 2014;163:88-101.

ABSTRACT FROM: Chowdhary N, Jotheeswaran AT, Nadkarni A, et al. The methods and outcomes of cultural adaptations of psychological treatments for depressive disorders: a systematic review. Psychol Med 2013;19:1-16.

Data sources Ovid Medline, EMBASE and PsycINFO searched from inception to December 2011, supplemented by hand search of reference lists.

Study type included RCTs and non-RCTs in adults with depression, that evaluated psychological treatments adapted for ethnic minorities.

Intervention Culturally adapted psychological treatments.

Comparison Enhanced care, usual care, antidepressants, no intervention or placebo.

\section{OUTCOMES}

Characteristics of included studies A total of 20 studies met inclusion criteria, 4 cluster RCTs, 14 RCTs and 2 non-RCTs $(n=4461)$. Nine of the studies included ethnic minorities in the US A (Latina, Puerto Rican, Hispanic, Chinese American and pregnant women of African American ethnicity), two in the UK (Black (not further defined), British Pakistani) and the remaining studies were conducted in Chile, Uganda, Jordan, Pakistan, India and Hong Kong. The included studies assessed culturally adapted Cognitive Behavioral Therapy $(n=10)$, interpersonal therapy $(n=4)$, psychoeducation $(n=3)$, problem-solving therapy $(n=2)$ and dynamic oriented therapy $(n=1)$.

Effect on depressive symptoms (primary outcome) Symptoms were assessed on nine different scales (most frequently the Beck Depression Inventory, $\mathrm{n}=5$ ) at follow-up times between 2 weeks and 18 months. Meta-analysis was conducted for 16 studies $(n=4162)$, which found that adapted psychological treatment improved depressive symptoms, compared to control, with a weighted standard mean difference of -0.72 ( $95 \%$ CI -0.94 to -0.49 ). 